Please quote as: Mauro, C.; Leimeister, J. M. \& Krcmar, H. (2011): The Nature of Medical Device Services. A Multiple-case Study. In: International Conference on Biomedical Electronics and Devices (BIODEVICES) 2011, Rome, Italy. 


\title{
THE NATURE OF MEDICAL DEVICE SERVICES A Multiple-Case Study
}

\author{
Christian Mauro, Helmut Krcmar \\ Information Systems, Technische Universität München, Boltzmannstr. 3, 85748 Garching, Germany \\ mauro@in.tum.de,krcmar@in.tum.de \\ Jan Marco Leimeister \\ Information Systems, Universität Kassel, Nora-Platiel-Str. 4, 34127 Kassel, Germany \\ leimeister@uni-kassel.de
}

\begin{abstract}
Keywords: $\quad$ Service Oriented Device Architecture, SODA, Service Oriented Architecture, SOA, Medical Devices.
Abstract: The integration of medical devices into the IT infrastructure of hospitals is still a major challenge. Recent research tries to adapt service oriented concepts to the field of medical devices in order to achieve an easier and better integration. However, no generalized framework for this kind of integration exists. The purpose of this paper is to identify the characteristics (the nature) of medical device services. The results were achieved by conducting a multiple-case study with three cases on two university hospitals. As a result, we identified twelve specific integration issues of medical devices and deduced eight characteristics. This paper contributes to the design of a generalized framework for the service oriented integration of medical devices. The identified characteristics can be interpreted as requirements to such a framework.
\end{abstract}

\section{INTRODUCTION}

The integration of medical devices into the IT infrastructure of hospitals is still an open issue (Lesh, Weininger, Goldman, Wilson, \& Himes, 2007). There is a huge gap between reality and the vision of seamless healthcare with horizontally and vertically integrated healthcare processes enabled by seamless IT support (Schweiger, A., Sunyaev, A., Leimeister, J. M., \& Krcmar, H., 2007). In order to bridge this gap several research projects are exploring the concept of service oriented device integration. The idea of this concept is the encapsulation of devices as services, analogous to enterprise services in service oriented architectures (SOA) (de Deugd, Carroll, Kelly, Millett, \& Ricker, 2006).

As we showed with a literature analysis, some first prototypical implementations achieved promising results (Mauro, C., Sunyaev, A., Leimeister, J. M., \& Krcmar, H., 2009). However, we also revealed that there is a lack of generalized concepts. In particular, a general framework for the service oriented integration of medical devices is missing.

Following a design science approach (according to Hevner, March, Park, and Ram (2004)), the aim of our research is to design, implement and evaluate such a framework. This paper presents an important step of this research, the exploration of the characteristics (the nature) of medical device services. This list of characteristics can be interpreted as requirements to the integration framework.

\section{SERVICE ORIENTED INTEGRATION OF LEGACY SYSTEMS AND DEVICES}

From an integration point of view, legacy systems and medical devices have some common characteristics (cf. Bennett (1995, p. 19), Bisbal, Lawless, Bing, \& Grimson (1999, p. 103), and Lesh, et al. (2007)):

- Slow hardware doesn't allow the use of modern software communication frameworks.

- A lack of clean interfaces complicates the integration with other systems.

- A difficult (or impossible) expandability doesn't allow modifications of insufficient interfaces. 
To overcome the integration issues of legacy systems a lot of publications and products are successfully using service oriented concepts (e.g., Siebenhaar, Lehrig, Braun, and Görge (2008)). In recent research, these concepts are adapted to devices (SODA, Service Oriented Device Architecture, cf. de Deugd et al. (2006)). Figure 1 shows a conceptual perspective view of the service oriented integration of devices. Following the contract-first principle (Erl, 2009), the starting point is the definition of the service contract followed by its implementation. An adapter component is necessary to handle the connection to the proprietary device interface. The strength of the concept is the loose coupling of service consumers to medical devices (Mauro, C., Leimeister, J. M., \& Krcmar, H., 2010). In this paper we use the term "medical device services" for services that encapsulate medical devices.

From a static point of view, the concept of the service oriented integration of legacy systems can directly be applied to medical devices. However, from a dynamic point of view we expect additional integration issues due to the characteristics of medical devices (e.g., mobility) and therefore of medical device services. With the following multiple-case study we try to identify the characteristics (the "nature") of medical device services.

\section{CASE STUDY DESIGN}

The multiple-case study is composed of three single cases conducted at two university hospitals (Figure 2 ). The cases were selected because of their different disciplines and our access to the field.

We use case studies for the exploration of the nature of medical device services due to the fact that different aspects (e.g., processes or the type of data provided by the devices) have to be considered. The handling of different kind of sources (observations, documents, interviews, etc.), as necessary for such an exploration, is a considerable strength of case studies (Yin, 2009, p. 11).

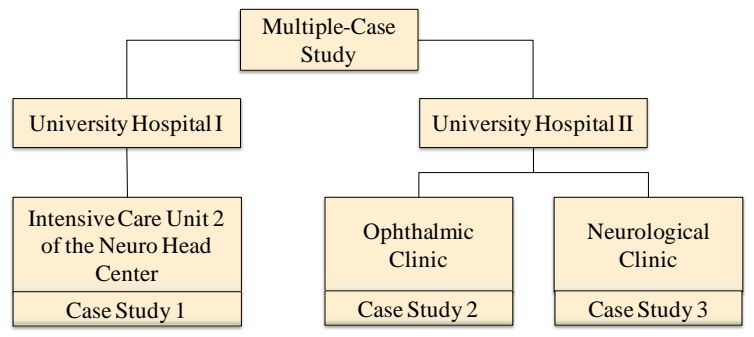

Figure 2: Multiple-case study with three cases.

Following Yin (2009, p. 27), the design of a case study consists of several elements. Table 1 shows these elements and how they are implemented in our case study. The strategy used for interpreting the data is implicit contained in the explanations of section 4 .

Table 1: Case study design.

\begin{tabular}{l}
$\begin{array}{l}\text { Purpose } \\
\text { Exploring the reasons for the complexity of the } \\
\text { integration of medical devices in order to draw } \\
\text { conclusions regarding the nature of medical device } \\
\text { services }\end{array}$ \\
\hline $\begin{array}{l}\text { Research Questions (RQ) } \\
\text { RQ1: How many systems might use the data provided } \\
\text { by medical devices? } \\
\text { RQ2: Which processes complicate the integration of } \\
\text { medical devices? } \\
\text { RQ3: Which legal restrictions complicate the } \\
\text { integration of medical devices? } \\
\text { RQ4: What kind of capabilities and data is provided by } \\
\text { the interfaces of medical devices? } \\
\text { RQ5: How are the grade of standardization and the } \\
\text { complexity of the interfaces of medical devices? }\end{array}$ \\
\hline $\begin{array}{l}\text { Units of analysis } \\
\text { All electronic medical devices of the respective } \\
\text { institution }\end{array}$
\end{tabular}

\section{Purpose}

conclusions regarding the nature of medical device services

Research Questions (RQ)

by medical devices?

Which processes complicate the integration of



Figure 1: Service oriented integration of devices (according to Mauro et al. (2010)). 


\begin{tabular}{|c|c|c|}
\hline Collected data and their link to the research questions \\
\hline RQ1, & $\begin{array}{c}\text { Processes using medical devices or data } \\
\text { provided by medical devices } \\
\text { (Source: Observation, interviews) }\end{array}$ \\
\hdashline RQ3 & $\begin{array}{c}\text { Regulatory } \\
\text { Framework } \\
\text { (Source: literature) }\end{array}$ & $\begin{array}{c}\text { Intended use of the } \\
\text { device interfaces } \\
\text { (Source: specificati- } \\
\text { ons and manuals) }\end{array}$ \\
\hdashline & $\begin{array}{c}\text { Complexity of } \\
\text { Rind of hardware } \\
\text { interfaces } \\
\text { (Source: on-site } \\
\text { analysis and } \\
\text { specifications }\end{array}$ & $\begin{array}{c}\text { (Source } \text { interfaces } \\
\text { specifications) }\end{array}$ \\
\hdashline $\begin{array}{c}\text { Kind of data } \\
\text { provided by } \\
\text { software interfaces } \\
\text { (Source: Protocol } \\
\text { specifications) }\end{array}$ & $\begin{array}{c}\text { Capabilities of } \\
\text { software interfaces } \\
\text { (Source: Protocol } \\
\text { specifications) }\end{array}$ \\
\hdashline $\begin{array}{c}\text { Grade of standardization of the software } \\
\text { interfaces } \\
\text { (Source: Protocol specifications) }\end{array}$ \\
\hline
\end{tabular}

Following the process proposed by Yin (2009, p. 57) we write an individual report for each case and present our cross-case conclusions in another section.

\section{CASE STUDY RESULTS}

\subsection{Case Study 1 - Intensive Care Unit}

\subsubsection{Medical devices and their interfaces}

At the Intensive Care Unit 2 (ICU2) the number of different devices is small. Basically, an intensive care bed is equipped with four types of devices: Infusion pumps (B. Braun Infusomat ${ }^{\circledR}$ Space), Syringe pumps (B. Braun Perfusor® Space), Respirator (Dräger Evita XL or Evita 4), and Patient monitor (GE Datex-Ohmeda).

B. Braun infusion and syringe pumps are inserted into a rack, the B. Braun SpaceStation. An optional module - the B. Braun SpaceCom - acts as a single access point for all pumps of a bed. This module has a number of standard hardware interfaces: Wireless LAN, Ethernet RJ45, PS/2 for barcode scanner, RS232 serial, and USB master/slave. Over these hardware interfaces three software interfaces are provided: a) A web server to configure the system and to access infusion data over a web browser, b) the medium complex proprietary BCC protocol, which can be used to access infusion data, and c) the simple XML based proprietary proposal data protocol, which can be used to send up to 24 medications to the pumps.

The Dräger Evita XL and Evita 4 respirators provide a serial RS-232 hardware interface for data export. The software interface is the proprietary and very complex Dräger MEDIBUS protocol. It supports a real time mode, which can be activated by using specific commands in order to support a continuous fast data flow.

Unfortunately, we didn't get access to the GE Datex-Ohmeda interface specification so far. Instead of that we analyzed the specification of a comparable patient monitor, the Dräger Infinity ${ }^{\circledR}$ Delta. This monitor provides two protocols: a) The medium complex proprietary RS-232 Export Protocol (via serial interface), which can be used to export several vital signs and alarms, and b) the medium complex proprietary Infinity ${ }^{\circledR}$ protocol (via LAN or wireless LAN), which provides monitoring data over network.

\subsubsection{Legal restrictions}

In Europe the Medical Device Directive is the most important legal basis. From an integration point of view, the two most important aspects are the upcoming norm IEC 80001, which defines regulations for the interconnection of medical devices, as well as the intended use of the device interface specified by the manufacturer (Mauro, C., Sunyaev, A., Dünnebeil, S., Leimeister, J. M., \& Krcmar, H., 2009). If the interface of a device is used in a way that is not intended by the manufacturer, the user (i.e., the hospital) is responsible for all resultant damages to the patient or other persons.

The intended use of all analyzed devices does not include the use of the provided data for diagnostic or therapeutic decisions. In addition, the SpaceCom specification prohibits a monitoring of alarms only on the basis of the data provided by the interface. These restrictions must be taken into consideration when connecting the devices to other systems.

\subsubsection{Process Analysis}

By observing the processes at the ICU2 we identified a number of (future) systems that could use the data provided by the medical devices: documentation systems (vital signs, care documentation, medication documentation, etc.), therapy planning systems, clinical trial systems, score calculation system (for the purpose of accounting a score value is calculated for each patient), IT supported ward rounds, and the medical technology database. We also identified a number of 
aspects that further complicate the integration of medical devices:

- Linking medical data to patients: Data provided by medical devices must be correctly linked to a patient. Linking data to the wrong patient could result in misinterpretations.

- Mobility of medical devices: Some devices are mobile in order to provide continuous care to the patient. As a consequence, devices may not be connected to the network at all times.

- Replacement of medical devices: If a device is defective (or needs to be maintained) it may dynamically be replaced by another one (other model, other manufacturer, etc.).

- Operation mode of medical devices: Devices can be switched on/off at any time, e.g., if a medication is stopped, the corresponding infusion pump may be switched off.

\subsubsection{Conclusion}

We conclude each case study with a reflection of the case study's research questions:

RQ1: Several systems (we identified six useful integration scenarios) may be interested in the data provided by medical devices (cf. section 4.1.3). RQ2: The linking of medical data to patients as well as the mobility and replacement of devices are critical aspects. Another challenge is the fact that medical devices may be switched on/off at any time (cf. section 4.1.3). RQ3: The upcoming norm IEC 80001 must be taken into consideration. In addition, the data provided by the analyzed medical devices must not be used for diagnostic or therapeutic decisions. In addition, the monitoring of alarms only on the basis of the data provided by the device interfaces is not allowed (cf. section 4.1.2). RQ4: The devices provide current vital signs and information about the current therapy by request or as continuous data flow. In addition, medications can be sent to the B. Braun pumps (cf. section 4.1.1). RQ5: The devices provide standardized hardware interfaces. However, all software interfaces are proprietary. The complexity of the protocols ranges from simple to very complex (cf. section 4.1.1).

\subsection{Case Study 2-Ophthalmic Clinic}

\subsubsection{Medical devices and their interfaces}

In this clinic we found 42 different (total number: 49) medical devices of 25 manufactures. Due to the great number of different devices we will not explain each device type in detail but summarize the most important results. Our analysis showed that the devices are of two kinds: a) Devices with no IT interface or with printer only (13), and b) devices that need special software (running on a internal or external computer) with a graphical user interface in order to analyze raw data and generate findings (29).

From an integration point of view, devices of the first category are quite unfavorable. There might be the possibility for some devices to redirect the printout into a file. However, e.g., for integrated thermal printer this is not possible at all. Devices of the second category cannot be directly integrated with other systems. Instead of that the corresponding software has to be integrated. Our analysis showed that about $50 \%$ of these software systems are supporting standards like DICOM (Digital Imaging and Communications in Medicine), GDT (Gerätedatentransfer, a common German standard) or HL7 (Health Level 7). The other half is providing proprietary interfaces and data formats.

Due to the specific discipline of the clinic the majority of the medical devices are producing image data. Therefore, the size of a dataset of one medical examination can be up to 200 megabyte.

\subsubsection{Legal restrictions}

We couldn't found any specific legal restrictions in the documents of the software systems. However, being software for the purpose of diagnostic the software itself might be (in future versions of the software: must be) a class IIa medical device due to the legal regulations (Mauro et al., 2009). Thus, the IEC 80001 must be taken into consideration when integrating such software systems (cf. section 4.1.2).

\subsubsection{Process Analysis}

The number of systems that may be interested in the data produced by the medical devices is very limited. In general, the generated findings are printed out and put into a paper-based medical record. Thus, after integrating the devices, the findings could directly be imported to an electronic medical record. Another system to be integrated may be the medical technology database (cf. section 4.1.3). Again, we identified a number of aspects that may complicate the integration of the devices:

- Linking medical data to patients: The linking of the data is not as difficult as in case study 1 . Administrative patient data (as name, gender, birthday, etc.) can be entered into the software. However, this manual step is error-prone, patient data may be entered incorrect.

- Operation mode of medical devices: This aspect 
is not as critical as in case study 1 because the software systems do not expect any requests. They may receive administrative patient data from the hospital information system, but such transfers are initiated by the user itself.

The mobility of medical devices is not relevant in case study 2 . Only three of the analyzed devices are used mobile. None of these devices has an IT interface, findings can only be printed. Also the replacement of a device could not be observed in this case study.

\subsubsection{Conclusion}

RQ1: Only a small number of systems (we identified two useful integration scenarios) may be interested in the data provided by medical devices (cf. section 4.2.3). $R Q 2$ : The administrative patient data should be imported from hospital information system to avoid manual mistakes. The fact that medical devices may be switched on/off at any time should be kept in mind (cf. section 4.2.3). RQ3: Medical software may be a medical device in the sense of the Medical Device Directive. In this case, the norm IEC 80001 has to be taken into consideration (cf. 4.2.2). RQ4: $31 \%$ of the analyzed device types do not provide an IT interface. The remaining $69 \%$ need special software in order to provide findings of an examination. This includes a textual report as well as different kind of data, e.g., numeric values or images up to $200 \mathrm{MB}$ (cf. 4.2.1). RQ5: The software interfaces are about $50 \%$ standardized and $50 \%$ proprietary. The complexity of the protocols ranges from simple to very complex (cf. 4.2.1).

\subsection{Case Study 3 - Neurological Clinic}

\subsubsection{Medical devices and their interfaces}

In this clinic we found 26 different (total number: 38) medical devices of 17 manufactures. The results are very similar to case study 2 . To avoid redundant explanations we will refer to case study 2 at some places.

Our analysis showed that all devices (except one device that only has printing capabilities) need special software (running on an internal or external computer) with a graphical user interface in order to analyze raw data and generate findings. Thus, as in case study 2, the devices cannot be directly integrated with other systems. Instead of that the corresponding software has to be integrated.

Again, about $50 \%$ of these software systems are supporting established standards. We also identified again a number of systems that produce a great amount of data (up to 400 megabyte when saving electrocardiogram video sequences).

\subsubsection{Legal restrictions}

Regarding legal restrictions our findings of case study 3 match with the findings of case study 2 .

\subsubsection{Process Analysis}

Regarding the process analysis our findings of case study 3 match with the findings of case study 2 . The only difference is that the mobility of the medical device is an important aspect. 15 of the analyzed medical devices are used mobile and therefore may be offline or appear in different network segments.

\subsubsection{Conclusion}

The results of case study 3 are very similar to the results of case study 2 . Thus, we only list the differences: a) In addition to images also video data up to $400 \mathrm{MB}$ are provided, b) only one of the analyzed device types does not provide an IT interface. All remaining devices need special software in order to provide findings of an examination, and c) $58 \%$ of the analyzed devices are used mobile.

\subsection{Cross-Case Conclusions}

In this section we provide cross-case conclusions in the form of a consolidated list of reasons for the complexity of the integration of medical devices. We compare these reasons with the integration issues of legacy systems (Table 2).

Table 2: Integration obstacles of medical devices.

\begin{tabular}{|c|l|c|}
\hline Nr. & Reason Description & LS \\
\hline 1 & $\begin{array}{l}\text { Several systems may have to be integrated } \\
\text { with the medical device. }\end{array}$ & X \\
\hline 2 & $\begin{array}{l}\text { Data provided by medical devices must be } \\
\text { correctly linked to a patient. }\end{array}$ & \\
\hline 3 & $\begin{array}{l}\text { Medical devices may be mobile and } \\
\text { therefore are not connected to the network } \\
\text { at all times. }\end{array}$ & $\begin{array}{l}\text { Medical devices (and with it the device's } \\
\text { hardware and software interfaces) may } \\
\text { dynamically be replaced by another one. }\end{array}$ \\
\hline 5 & $\begin{array}{l}\text { Medical devices can be switched on/off at } \\
\text { any time. }\end{array}$ & \\
\hline 6 & $\begin{array}{l}\text { Due to legal restrictions the interface of a } \\
\text { medical device cannot be changed. }\end{array}$ & $\mathrm{X}$ \\
\hline 7 & A risk management (IEC 80001) must be & \\
\hline
\end{tabular}




\begin{tabular}{|c|l|c|}
\hline & $\begin{array}{l}\text { conducted when connecting medical } \\
\text { devices with other systems. }\end{array}$ & \\
\hline 8 & $\begin{array}{l}\text { The interface of a medical device is only } \\
\text { allowed to be used as intended by the } \\
\text { manufacturer. }\end{array}$ & \\
\hline 9 & $\begin{array}{l}\text { Medical devices may provide streaming } \\
\text { data. }\end{array}$ & $\mathrm{X}$ \\
\hline 10 & $\begin{array}{l}\text { Medical devices may provide huge image } \\
\text { or video files. }\end{array}$ & $\mathrm{X}$ \\
\hline 11 & $\begin{array}{l}\text { Medical devices often provide proprietary } \\
\text { and complex interfaces. }\end{array}$ & $\mathrm{X}$ \\
\hline 12 & $\begin{array}{l}\text { Medical devices do not provide modern } \\
\text { communication frameworks. }\end{array}$ \\
\hline $\begin{array}{l}\mathrm{Nr} .=\text { Reason Number, LS = Legacy System, } \\
\mathrm{X}=\text { Analogous also valid for legacy systems }\end{array}$ \\
\hline
\end{tabular}

\section{DEDUCING THE NATURE OF MEDICAL DEVICE SERVICES}

Based on the identified reasons presented in the previous section we deduced the characteristics of medical device services. The following list presents the characteristics as well as the references to the reasons they were deduced from. Medical device services

- correctly link their medical data to patients (Reason 2),

- dynamically appear and disappear at the network (Reasons 3, 4, and 5),

- automatically publish themselves (as a consequence of Reasons 3, 4 and 5),

- dynamically communicate with different hardware interfaces and complex low-level device interfaces (Reasons 4, 6, 11, and 12),

- have passed a IEC 80001 risk management process (Reason 7),

- respect the intended use of the device interfaces (Reason 8),

- may provide streaming data (Reason 9), and

- may provide huge amount of data (Reason 10).

Reason 1 doesn't result in an item of this list. Services are intended to be used by several other systems so this aspect is not a characteristic of medical device services.

\section{CONCLUSION AND FUTURE RESEARCH}

In this paper we presented the nature of medical device services in the form of a list of eight characteristics. The results were deduced by conducting a multiple-case study comprised of three cases at two university hospitals. This list of characteristics can be considered as a list of requirements to a service oriented integration framework for medical devices. Such a concept must support / realize all items of the list. Thus, our next step will be designing a service oriented integration framework for medical devices based on existing SOA design patterns / SOA best practices. Finally, we will implement and evaluate the created framework.

\section{REFERENCES}

Bennett, K. (1995). Legacy Systems: Coping with Success. IEEE Software, 12(1), 19-23.

Bisbal, J., Lawless, D., Bing, W., \& Grimson, J. (1999). Legacy information systems: issues and directions. IEEE Software, 16(5), 103-111.

de Deugd, S., Carroll, R., Kelly, K. E., Millett, B., \& Ricker, J. (2006). SODA: Service Oriented Device Architecture. Pervasive Computing, IEEE, 5(3), 9496.

Erl, T. (2009). SOA Design Patterns. Boston: Prentice Hall International.

Hevner, A. R., March, S. T., Park, J., \& Ram, S. (2004). Design Science in Information Systems Research. MIS Quarterly, 28(1), 75-105.

Lesh, K., Weininger, S., Goldman, J. M., Wilson, B., \& Himes, G. (2007). Medical Device Interoperability Assessing the Environment. Paper presented at the Joint Workshop on High Confidence Medical Devices, Software, and Systems and Medical Device Plug-andPlay Interoperability.

Mauro, C., Leimeister, J. M., \& Krcmar, H. (2010). Serviceorientierte Integration medizinischer Geräte ganzheitliche IT-Unterstützung klinischer Prozesse. Informatik-Spektrum, Online First.

Mauro, C., Sunyaev, A., Leimeister, J. M., \& Krcmar, H. (2009). Service-orientierte Integration medizinischer Geräte - eine State of the Art Analyse. Paper presented at the Wirtschaftsinformatik 2009 - Business Services: Konzepte, Technologien und Anwendungen.

Mauro, C., Sunyaev, A., Dünnebeil, S., Leimeister, J. M., \& Krcmar, H. (2009). Mobile Anwendungen im Kontext des Medizinproduktegesetzes. Paper presented at the Informatik 2009 - Im Focus das Leben.

Schweiger, A., Sunyaev, A., Leimeister, J. M., \& Krcmar, H. (2007). Toward Seamless Healthcare with Software Agents. Communications of the Association for Information Systems (CAIS), 19, 692-709.

Siebenhaar, M., Lehrig, T., Braun, J., \& Görge, T. (2008). Entwicklung einer SOA-basierten Webanwendung zur Buchung und Verwaltung von Segeltouren: Proprietäre Software vs. Open Source Wirtschaftsinformatik, 50(4), 325-329.

Yin, R. K. (2009). Case study research: design and methods (4 ed.). California: SAGE Publications. 